\title{
Assessing the Operator's Readiness to Perform Tasks of Controlling by the Unmanned Aerial Platforms
}

\author{
Dmytro Kucherov ${ }^{1, *}$, Olha Sushchenko ${ }^{2}$, Andrii Kozub $^{3}$, Volodymyr Nakonechnyi ${ }^{4}$ \\ ${ }^{1}$ Department of Computerized Control Systems, National Aviation University, Kyiv, 03058, Ukraine \\ ${ }^{2}$ Department of Aerospace Control Systems, Institute, National Aviation University, Kyiv, 03058, Ukraine \\ ${ }^{3}$ National Space Facilities Control and Test Center, State Space Agency, Kyiv, 01010, Ukraine \\ ${ }^{4}$ Department of Cyber Security and Information Protection, Taras Shevchenko National University, Kyiv, 01033, Ukraine
}

\begin{tabular}{l} 
A R T I C L E I N F O \\
\hline Article history: \\
Received: 09 July, 2020 \\
Accepted: 19 July, 2020 \\
Online: 09 August, 2020 \\
\hline Keywords: \\
Operator of unmanned aerial \\
platform \\
Assessment criteria \\
Quality of operator activity \\
Method of hierarchical analysis
\end{tabular}

\section{Introduction}

Modern successes in the field of microelectronics and electromechanics have made it possible to create unmanned aerial vehicles that are effectively used to solve various problems [1]. The control of an unmanned vehicle can be performed manually, automatically and semi-automatically, where the role of the human as the machine operator is very important both in preparing and conducting the flight. The operator's remoteness from the control means creates several difficulties in flight control, primarily related to control delay, inadequate presentation of information about the environment, and the dynamics of the control process itself.

\footnotetext{
${ }^{*}$ Corresponding Author: Dmytro Kucherov, Dept. of Computerized Control
} Systems, Faculty of Cybersecurity, Computer and Software Engineering, National Aviation University, Kyiv, Ukraine, d kucherov@ukr.net
A typical flight control system includes an operator, on-board receiving and control equipment, and a ground-based transmitting station, usually a tablet computer with a software interface, and the aircraft itself. The control of a military unmanned vehicle usually involves two human operators, one of them is responsible for controlling the movement of the vehicle (aircraft operator), and the second ensures the fulfillment of the target task (operator of the target task load). Therefore, the management of an unmanned aerial platform is not a simple task but requires training the operator in skills that will help him cope with the control difficulties in conditions of natural and artificial interference, loss of a communication channel, loss of line of sight, delays and distortions of transmitted information, control of many devices, etc. 
Completing the target at the terminal point requires significantly more time to prepare the platform for flight in automatic than in manual or semi-automatic modes. These conditions imply the fulfillment of many requirements for the practical skills of operators that can ensure the implementation of the target in real flight conditions.

Our contribution consists of developing the versatile method's assessment of the productivity of operators based on measurement of their indicators such as reaction time, the accuracy of the operation, stress resistance, the fitness of mind and hands, preparedness, and foresight. The proposed method is suitable not only for assessing the productivity of UAV operators but operators of some other industries, where high requirements imposed on the organization of labor.

The rest paper is organized as follows: a literature review is given in the next section, the control system structure and typical operator actions are presented in Section 3, the problem statement is given in Section 4, the solution method is presented in Section 5 , numerical examples and discussion of input data are given in Section 6, and conclusions are discussed in Section 7.

\section{Review of Previous Researches}

This work is an extended version of the report [1] presented at the 5th International Conference Actual Problems of Unmanned Aerial Vehicles Developments (APUAVD), where a method for evaluating the operator's activity taking into account the study of his psycho-physical reactions is proposed.

An analytical review of the influence of the human factor on the efficiency of using unmanned aerial vehicles (UAVs) in the current and immediate future is presented in [2]. The study emphasizes the need for special training by the UAV human operator, which, in principle, differs from that to which the pilots are involved. The review also notes the need for additional scientific research regarding the skills, knowledge, and abilities of UAV operators and ways to evaluate them.

In [3], the importance of the influence of a human operator on the efficiency and quality of control of unmanned aerial vehicles is noted. The paper notes that about $70 \%$ of all UAV-related accidents are caused by erroneous human activities. Despite the high level of automation, a person is not excluded from the operation of the control system, but rather, on the contrary, takes a leading place in the preparation, conduct, and implementation of tasks, eliminating the risks associated with the death of the pilot. The presence of several operators involves the coordination of their actions and interaction to achieve the ultimate goal of using one or a group of devices.

In [4-8], factors are considered that affect the preparation and quality of exploitation of unmanned platforms by operators. So, the effect of spatial capabilities obtained by the operator through the use of UAVs was evaluated in [4]. A sense of space gives confidence to the operator when acting in whichever situation at any time of the day and for any type of purpose.

In [5], a simulator description is proposed for a UAV operator with wide simulation capabilities, namely: flight control, wireless communications, and ground equipment. However, the article does not provide the parameters and methods for evaluating the operator.

In [6], the UAV operator errors were presented in the form of a chi-square curve of the Pearson distribution, obtained by constructing a histogram of the probability density of measurements of errors in operator activity.

The authors of [7] propose evaluating the capabilities of the UAV operator according to the profile generated by special software. As estimates, metrics are proposed that are based on a combination of fuzzy logic methods and cluster analysis. The proposed techniques allow us to evaluate the operator not only of a single device but when managing a group.

Errors of a technical nature were analyzed in [8]. It indicates the technical problems that the operator encounters, namely, the limited field of view, delays in the transfer of information, poor operator understanding of the situation in which the unmanned vehicle is located, and errors on-board equipment.

The methodical complex for accelerated training of UAV operators is presented in [9], the components of which are control situations with corresponding difficulties and the intensity of their implementation for a certain time. It is assumed that during the preparation of the operator by this complex, the generalized indicator of operator readiness assumes some steady-state value.

In the study the authors of [10] the temporal norms of operator training for the formation of the required psycho-physiological characteristics of personnel for unmanned aviation systems. The study relies on training programs for the air force. An assessment of the quality of preparedness of operators and methods for their certification in the study is not considered.

The usefulness of practical developments aimed at training operators who are ready to work in conditions when the equipment is on the verge of failure is proposed in [11].

In [12], a system for modeling dynamics is given, which allows one to analyze factors affecting the training of the professional skills of coal mine operators. The work explores operator training over a long time. Also, priority is determined, an important place among which is the ability to work in difficult conditions.

For some industries, it is important to maintain operator productivity in conditions when the order of actions changes [13, 14]. In this case, there is a need to redistribute the procedure and change the training schedule, and then it is useful a loss training procedure is proposed. The authors also introduced an indicator of operator experience [13]. The skill indicator in the study is represented by a multi-purpose function that takes into account the skill indicator and working time. In research [14] proposes an additional indicator, the standardization point.

The Italian concern Terna [15] has developed three main programs for training and certification of operators in real-time: the initial program, sliding (continuous) training, and the general program for other interested parties. The training is configured for the specific role of each operator in real-time and covers all its operational activities. Besides, an operating training simulator (OTS) has been developed by the company. 
The authors of [16] evaluated the mental workload of operators whose main professional activity is air traffic control and students by comparing continuous exposure close to the infrared range and a mobile touch panel of the same range worn on the operator's head. The study revealed the possibility of using a flexible touch panel to assess the mental workload of operators involved in air traffic control based on the repetition of measurements and the ANOVA technique.

The work [17] presents a methodology for the development of psychomotor skills through the use of teaching means. The results of the study showed a positive influence on the development of psychomotor skills in people of different categories.

The article [18] considers the assessment of human hearing by methods by a system of clinical audiometry. A biotechnological system for assessment and stimulating the human auditory analyzer under the influence of various factors was developed and tested, which allowed us to study the effect of sound frequencies on the patient.

The method of training a group of agents is presented in [19]. Training is based on the introduction of a Q-function, the result of which depends on the joint actions of a pair of agents. Unfortunately, the result is characterized by the limited elements involved in the learning process, and simple scenarios are considered.

In [20], [21], the UAV control system is studied, which can become the basis for the construction of a simulator designed for training, preparing, and certification of operators.

Based on the papers review, we can conclude that there is strong usefulness in developing and implement appropriate methods that will make the operator certification process more accurate.

\section{Features of the Controlling of Unmanned Platform}

The section briefly discusses the generalized structural composition of a typical unmanned platform, the main problems of flight control, and the requirements for operator actions.

\subsection{System structure}

The structure of an automated UAV control system with a human operator in the control loop has the form shown in Figure 1. The operator's workplace is a remote control that provides several controls that are available on the display screen.

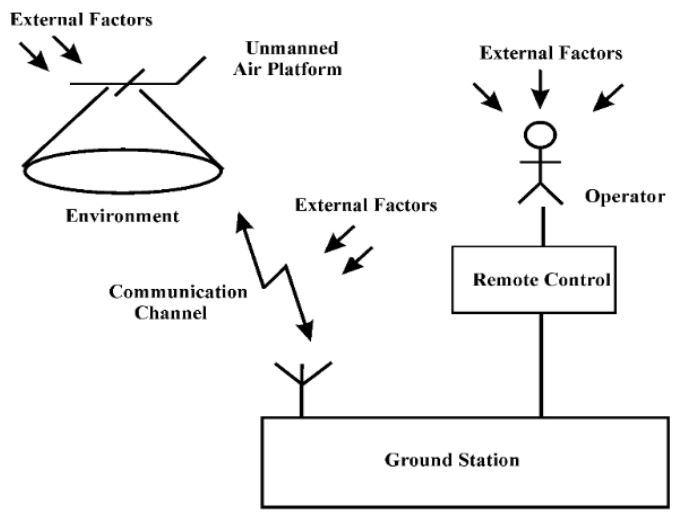

Figure 1: The structure of an automated control system of UAV www.astesj.com

\subsection{Flight Control Problems}

A vulnerable place to control the UAV platform is the flight control channel. The problem here is the limited bandwidth of the radio control channel since the narrow-band path is considered susceptible to interfering signals that occur in the radio channel. In-flight, communication losses are also possible; in places with strong reflections, erroneous or false signals and radio control commands can be received. Besides, the complexity of radio command control lies in the low data rate.

\subsection{Operator Actions}

The operator controlling the flight of the UAV must know the modes and parameters of the control channel that ensure a stable flight of the platform; be able to make adjustments to flight control; know the conditions for switching manual and semi-automatic modes to autopilot; be prepared to exit autopilot with appropriate authorization.

The operator responsible for carrying out the useful task should be well aware of the capabilities of the equipment used, be able to control existing cameras, be able to extract useful information from the received data, and also closely cooperate with the flight control operator.

\section{Problem Statement}

Long-term work with the console puts forward some specific requirements for the operator. The situation displayed on the remote control screen is similar to a computer game, and therefore the operator's actions are similar to the reactions of an experienced gamer. The main ones among them are the reaction to the event, its accuracy, perception, and understanding of the displayed situation with a certain delay, fitness of mind and hands, characterized by resistance to long work; stress resistance, determined by the degree of resistance to negative influences and unexpected situations; foresight is the possibility of seeing or feeling probable future events that may occur.

The following performance indicators of the operator are offered:

- the reaction time $t$ is considered the main parameter characterizing the behavior of the operator; it is determined by the time the irritant occurs before the reaction to the event;

- the accuracy of the operation $\varepsilon$, which is the degree to which the data read by the operator corresponds to the values read from the screen;

- exposure $\tau$ is the time the operator understands the displayed situation;

- fitness of mind and hands $v$, characterized by the frequency of errors that occur in the work of the operator;

- stress resistance $k$ shows the degree of resistance to negative impacts of pressure factors;

- preparedness $\Sigma$ is a set of knowledge, skills, and conditions of mental and physiological functions that establish its ability to perform specified actions with a certain quality;

- foresight $\lambda$ is the ability to see or feel the possible future that will take place soon. 
The analysis of the selected indicators shows their heterogeneity. There are time indicators estimated by absolute values, this is the reaction time $t$ and the exposure time $\tau$, measured in seconds. The frequency of errors $v$, we will measure in relative units, while $v \leq v_{\max } \leq 1$, the value $v_{\max }$ corresponds to an unacceptable level of errors. In the interest of generality, we write $v_{\min } \leq v \leq v_{\max }$, where the desired value is $v_{\min } \approx 0$. Stress tolerance $k$, preparedness $\Sigma$, and foresight $\lambda$ should be evaluated in scores. The accuracy of the operation $\varepsilon$ when using, for example, a stylus or other manipulator, we will evaluate the distance between the true value and the exact coordinates, in the scale of the display screen this value is measured in millimeters.

Assuming that the real indicators of the operator can be in some intervals allowed by various standards, we will suggest that the measured parameter is in the intervals

$$
\underline{\xi} \leq \xi_{i} \leq \bar{\xi},
$$

where $\underline{\xi}, \bar{\xi}$ are the minimum and maximum values of the measured parameters, and $i=1 . . N, N$ is the number of indicators.

The heterogeneity of the given parameters is also manifested in the inconsistency of the values of the indicators. For example, the values of reaction time $t$, accuracy $\varepsilon$, the fitness of mind and hands $v$ should be small, while exposure $\tau$, stress resistance $k$, preparedness $\Sigma$, and foresight $\lambda$ for a good operator should correspond to higher values in their ranges.

It is proposed to evaluate the generalized indicator of operator suitability by the relation

$$
J\left(\xi_{i} \mid i=1 \ldots N\right) \geq J_{\min },
$$

where $J_{\min }$ is a certain threshold value of the indicator by which a decision is made on the operator's admission to the activity. We will call the $J$ as the performance of the operator.

The task is to find a method for determining the value $J_{\min }$ of expression (2), in the presence of measured parameters $\xi_{i}$ satisfying inequality (1), which will allow us to establish inequality (2), which will give an objective assessment of the operator's readiness to carry out the task of controlling an unmanned platform taking into account the influence heterogeneities $\xi_{i}$.

\section{Technique of Solving}

The section discusses the approach to calculating the criterion and the methodology for calculating operator productivity.

\subsection{Operator Assessment Criteria}

The operator's productivity estimation based on the introduced set of indicators will be calculated in the form of a linear function that written as follow

$$
J=\sum_{i=1}^{N} a_{i} \sigma_{i}
$$

where $a_{i}$ is the $i$ th weight coefficient, $\sigma_{i}$ is the $i$ th normalized parameter, i.e.

$$
\sigma_{i}=\frac{\bar{\xi}-\xi_{i}}{\bar{\xi}-\underline{\xi}}
$$

where $\xi_{i}$ is the measured value of the parameter, $i=1, \ldots, N$. The restriction is imposed on the weighting coefficients

$$
\sum_{i=1}^{N} a_{i}=1
$$

Now the task of assessing the quality of operator training is reduced to determining the value of $J_{\min }$ with unknown weights $a_{i}$.

Since the introduced indicators have different effects on the value of $J_{m i n}$, and for the most part are contradictory, for example, indicators such as reaction time t, accuracy (error) $\varepsilon$ and fitness $v$ must be small and the others should be as large as possible, it makes sense to separate these factors into two opposite groups, and solve the maximization problem taking into consideration the equal contribution of each component, i.e. define a threshold value as

$$
J_{\min }=\max _{a_{i}}\left(\frac{1}{m_{1}} \sum_{i=1}^{m_{1}} a_{i} \sigma_{i}^{+}+\frac{1}{m_{2}} \sum_{i=m_{1}+1}^{m_{2}} a_{i} \sigma_{i}^{-}\right),
$$

where $m_{1}+m_{2}=N$, in the case the considered parameters $N=7$.

Expressions (3), (4), (6), and taking into account (1) - (5) as well, provide finding the threshold $J_{\min }$.

\subsection{Calculation Approach}

The computational procedure involves this sequence of steps

1. Measured are the values of the parameter vector $\xi$ satisfying inequality (1).

2. We calculate the values of the coefficients $a_{i}$ by the method of hierarchical analysis, including the preparation of the matrix $A$ of pairwise comparisons of size $p \times p$, where $p$ is the number of criteria. We set the diagonal elements of the matrix $A$ equal to 1 , calculate the values of the elements $a_{i j}$, taking into account the importance of the measured parameter so that the parameter takes a value in the range, for example, from 1 to 9 . In case, when we deal with the most important parameter it takes the value 9 , otherwise the least important will be 1 . The element $a_{j i}=1 / a_{i j}$.

3. Next, the fulfillment of the consistency condition for the matrix of pairwise comparisons is checked by calculating the consistency coefficient CR

$$
C C=\frac{p\left(p_{\max }-p\right)}{1.98(p-1)(p-2)} .
$$

If $C C \leq 0.1$, the level of consistency of matrix $A$ is considered acceptable; otherwise, matrix $A$ is checked.

4. Determine the value of $J_{\min }(6)$. 
5. We measure the parameters of the vector $\xi$ for a particular human operator, apply (2), (1) and (4).

Corollary 1 . The quantity $J$ satisfies the condition $0<J \leq$ $J_{\max }$.

Corollary 2. The value $J_{\max }=1$.

\section{Simulation}

An important point in modeling is the selection of suitable parameters and the evaluation of the results. These issues are addressed in this section.

\subsection{Some information about parameter values}

The reaction time of the operator is determined by the delay times of the signal $t_{i}$ in the $i$ th control device and the delay of the signal from the moment the operator recognizes the situation to the corresponding reaction $t_{d e l}$, or

$$
t=\sum_{i=1}^{n} t_{i}+t_{d e l}
$$

where $n$ is the total number of controls. The delay entered by the operator far exceeds the control delay of the computer. This value is in the range of $100 \ldots 500 \mathrm{~ms}$, which corresponds to the reaction of the gamer. The best indicator is the smaller value.

The exposure time $\tau$ of the operator's eye is also determined by two factors: the ability to notice a new luminous object and the time of awareness of this object. A trained eye is capable of detecting flashes of light lasting $5-10 \mathrm{~ms}$, however, this parameter depends on the surrounding light. American scientist B. Libet found that conscious human activity manifests itself after 300-500 $\mathrm{ms}$ of the fact that he decided to move his hand [22].

The accuracy $\varepsilon$ of the operator's actions is determined by the thickness of the user device and the size of the spot on the screen, which the operator must reliably overlap by the device used. The operator's action will be performed in case of a $50 \%$ capture of the screen spot with the stylus tip. For example, if the diameter of the stylus tip is $1.9-2 \mathrm{~mm}$, then approximately $1 \mathrm{~mm}$ is the accuracy of the operator [23].

The level of operator errors $v$ depends on the degree of preparedness, workload, and fatigue due to long work. According to experimental data obtained during the processing of visual signals [24], the following results were obtained: for beginners who do not have the experience, the error rate is 0.27 , for operators who have completed 4 or more training, this indicator is 0.018 . Since there are, as a rule, no untrained operators, the maximum value of the indicator can be taken as 0.15 .

Indicators evaluated on a scale of points, which include stress resistance $k$, preparedness $\Sigma$, foresight $\lambda$, can also be assessed on a categorical scale in terms: excellent, good, satisfactory, poor. Having chosen this scale as a basis, it is possible to make such a correspondence. The category "excellent" is estimated at 8-9 scores, "good" - 5-7 scores, "satisfactory" - 3-4 scores, "poor" - 12 scores.

\section{2. $\quad$ Numerical examples}

The initial data accepted as the norm are given in Table I. Following the procedure described in the solution section and formulas (3), (4), (6) for $N=7$, we found weight coefficients that satisfy the consistency condition for the initial matrices of pairwise comparisons (Table 1).

Table 1: Normal Data

\begin{tabular}{rl|l|l|l|l|l|l|l|}
\hline $\begin{array}{l}\text { para } \\
\text { meter }\end{array}$ & $\underline{t}$ & $\underline{\varepsilon}$ & $\underline{\tau}$ & $\underline{v}$ & $\bar{k}$ & $\bar{\Sigma}$ & $\bar{\lambda}$ \\
\hline value & $.25 \mathrm{~s}$ & $\begin{array}{l}.9 \\
\mathrm{~mm}\end{array}$ & $.4 \mathrm{~s}$ & .07 & 3 & 3 & 2 \\
\hline$\sigma_{i}$ & .625 & .5 & .5 & .6 & .25 & .25 & .25 \\
\hline & $a_{\underline{t}}=0.1017, a_{\underline{\varepsilon}}=0.1835, a_{\underline{\tau}}=0.2602$, \\
$a_{\underline{v}}$ & $=0.4545, a_{\bar{k}}=0.5972, a_{\bar{\Sigma}}=0.2847, a_{\bar{\lambda}}=0.1181$
\end{tabular}

Using the hierarchy analysis method [25], weights were determined that satisfy the consistency condition for the initial matrices of pairwise comparisons $A$. Then, following (6), we obtained the value $J_{\min }=0.2788$. It should be noted that condition (5) is only satisfied with the components of expression (6), enclosed in brackets.

Now we will check the weights for other, obviously worse values of the considered parameters, for example, for the data in Table 2.

Table 2: The Second Set of Data

\begin{tabular}{|l|l|l|l|l|l|l|l|}
\hline $\begin{array}{l}\text { para } \\
\text { meter }\end{array}$ & $t$ & $\varepsilon$ & $\tau$ & $v$ & $k$ & $\Sigma$ & $\lambda$ \\
\hline Value & $.6 \mathrm{~s}$ & $\begin{array}{l}1.5 \\
\mathrm{~mm}\end{array}$ & $.55 \mathrm{~s}$ & .2 & 2 & 2 & 2 \\
\hline$\sigma_{i}$ & -.25 & -2.5 & -.25 & -.38 & .88 & .88 & .88 \\
\hline
\end{tabular}

We obtain the value $J=0.1536$, which does not satisfy condition (2).

Now consider the best indicators than in Table 2 and evaluated these results, Table 3.

Table 3: The Third Set of Data

\begin{tabular}{|l|l|l|l|l|l|l|l|}
\hline $\begin{array}{l}\text { para } \\
\text { meter }\end{array}$ & $t$ & $\varepsilon$ & $\tau$ & $\nu$ & $k$ & $\Sigma$ & $\lambda$ \\
\hline Value & .2 & .85 & .35 & .05 & 8 & 8 & 8 \\
\hline$\sigma_{i}$ & .75 & .75 & .75 & .76 & .125 & .125 & .125 \\
\hline
\end{tabular}

We obtain the value $J=0.8783$, which fully satisfies condition (11). The results obtained completely coincide with the proposals put forward, and also confirm the study conducted in [26]. Also, we expect that the developed methodology turns out to be enough effective in practical application with any set of measurement indicators.

\section{Conclusions}

A study of the expansion of research on unmanned platforms confirms the need for special training of operators for maintenance, operation and the performance of tasks as intended. 
The paper proposes an approach based on the entering of a generalized indicator of the operator's productivity, which is a linear function of partial heterogeneous quality criteria with unknown weight coefficients. The described method is a modification of the method for analyzing process hierarchies, based on the identification of indicators of the same type, the effectiveness of which is confirmed by numerical examples. The considered approach can be used in the certification of the operator after passing the training process. Our further research we will plan to be directed to the development and implementation of software for the simulator complex with assessing the training of UAV operators following the developed methodology.

\section{Conflict of Interest}

The authors declare no conflict of interest.

\section{Acknowledgment}

The authors thank both the authorities of the State Space Agency of Ukraine, Taras Shevchenko National University, and National Aviation University the especially leadership of the Faculty of Cybersecurity, Computer and Software Engineering for their support during the preparation of this paper. The authors also thank the anonymous reviewers, whose comments significantly improved the content of the paper.

\section{References}

[1] D. Kucherov, O. Sushchenko, A. Kozub, "Operator Training for Unmanned Aerial Vehicles Control" in 2019 IEEE 5th International Conference Actual Problems of Unmanned Aerial Vehicles Developments (APUAVD), 22-24 Oct., Kiev, Ukraine, 1-4, 2019. https://doi.org/ 10.1109/APUAVD47061.2019.8943918

[2] J. S. McCarley, C.D. Wickens, "Human Factors Implications of UAVs in the National Airspace", $2019.2 \quad$ https://www.researchgate .net/publication/228358350_Human_factors_implications_of_UAVs_ in_the_national_airspace, last accessed 2019/03/13.

[3] R. M. Taylor, "Human Automation Integration for Supervisory Control of UAVs" in Virtual Media for Military Applications, RTO-MP-HFM-136, 12$1-12-10,2006$.

[4] J. Y. C. Chen, "Effects of operator spatial ability on UAV-guided ground navigation" in 5th ACM/IEEE International Conference on Human-Robot Interaction (HRI), 2-5 March 2010, Osaka, Japan, Proceedings, 139 - 140, 2010. https://doi.org/10.1109/HRI.2010.5453227

[5] J. Wu, W. Wang, J. Zhang, B. Wang, "Research of a kind of new UAV training simulator based on equipment simulation" in 2011 International Conference on Electronic \& Mechanical Engineering and Information Technology, 12-14 Aug., Harbin, China, Proceedings, 4812 - 4815, 2011. https://doi.org/10.1109/EMEIT.2011.6024116

[6] O. V. Kozhokhina, V. M. Gribov, L. V. Blahaia, "Processing statistical data about UAV operator errors", in IEEE 3rd International Conference Actual Problems of Unmanned Aerial Vehicles Developments (APUAVD), 13-15 October, Kyiv, Ukraine, Proceedings, $124-127,2015$. https://doi.org/10.1109/APUAVD.2015.7346578

[7] V. Rodriguez-Fernandez, H.D. Menendez, D. Camacho, "Automatic profile generation for UAV operators using a simulation-based training environment", Lect. Notes Artif. Int., 5 (1), 37 - 46, 2016. https://doi.org/10.1007/s13748-015-0072-y

[8] K. W. Williams, "Human Factors Implications of Unmanned Aircraft Accidents: Flight-Control Problems", Federal Aviation Administration, April, $1-6$, 2006. DOT/FAA/AM-06/8. https://doi.org/10.1016/S14793601(05)07008-6.

[9] Y. A. Gunchenko, S. A. Shvorov, N. D. Rudnichenko, V. D. Boyko, "Methodical complex of accelerated training for operators of unmanned aerial vehicles" in 2016 4th International Conference on Methods and Systems of Navigation and Motion Control (MSNMC), 18-20 Oct., Kiev, Ukraine, 130133, 2016. https://doi.org/10.1109/MSNMC.2016.7783124
[10] S. M. Zlepko, L. G. Koval, D. Kh. Shtofel, V. O. Homolinskyi, M. I. Palamarchuk," Peculiarities of Psycho-Physiological Selection of Operators for Unmanned Aviation Systems" in 2020 IEEE 15th International Conference on Advanced Trends in Radioelectronics, Telecommunications and Computer Engineering (TCSET), 25-29 Feb., Lviv-Slavske, Ukraine, Ukraine, 628-631, 2020. https://doi.org/10.1109/TCSET49122.2020.235508

[11] J. Dagle, ""Partial panel" operator training: advanced simulator training to enhance situational awareness in off-normal situations" in 2006 IEEE Power Engineering Society General Meeting, 18-22 June, Montreal, Que., Canada, 1-2, 2006. https://doi.org/10.1109/PES.2006.1709229

[12] D. Hong-bin, G. Tao-li, D. Xiao-li, "A System Dynamics simulation analysis of the Influencing Factors of coalmine special operators' competency by training" in 2nd IEEE International Conference on Information Management and Engineering, 16-18 April, Chengdu, China, 367-370, 2010. https://doi.org/ 10.1109/ICIME.2010.5477580

[13] H. Haraguchi, T. Kaihara, N. Fujii, D. Kokuryo, "A study of designing for the operator training under an order change in cell manufacturing system" in 55th Annual Conference of the Society of Instrument and Control Engineers of Japan (SICE), 20-23 Sept., Tsukuba, Japan, 802-806, 2016. https://doi.org/10.1109/SICE.2016.7749216

[14] H. Haraguchi, "A Study on Operator Allocation Method Considering the Productivity and the Training Effect in Labor-Intensive Manufacturing System" in 2019 IEEE International Conference on Industrial Engineering and Engineering Management (IEEM), 15-18 Dec., Macao, Macao, Macao, 1236 - 1239, 2019. https://doi.org/10.1109/IEEM44572.2019.8978613

[15] C. Coluzzi, M. Contu, G. Pecoraro, S. Orlandi, F. Allella, A. Pascucci, S. Moroni, "Real time operators training: Terna experience in implementing the Commission Regulation (EU) 2017/1485" AEIT International Annual Conference (AEIT), 18-20 Sept. 2019, Florence, Italy, Italy, p. 1 - 5, 2019. https://doi.org/10.23919/AEIT.2019.8893413

[16] H. Ayaz, P. A. Shewokis, S. Bunce, K. Izzetoglu, B. Willems, B. Onaral, "Optical brain monitoring for operator training and mental workload assessment", NeuroImage 59, 36-47, 2012. https://doi.org/10.1016/ j.neuroimage.2011.06.023

[17] K.G. Selivanova, O.V. Ignashchuk, L.G.Koval et al. "Computer-aided system for interactive psychomotor testing," Photonics Applications in Astronomy, Communications, Industry, and High Energy Physics Experiments, Proc. SPIE, vol. 10445, 2017. https://doi.org/10.1117/12.2280815.

[18] M.V. Bachynskiy, O.Yu. Azarkhov, D.Kh. Shtofel et al. "System and algorithm for evaluation of human auditory analyzer state," Photonics Applications in Astronomy, Communications, Industry, and High Energy Physics Experiments, Proc. SPIE, vol. 10445, 2017. https://doi.org/ 10.1117/12.2280738.

[19] Wiem Zemzem, Ines Hosni, “A New Distributed Reinforcement Learning Approach for Multiagent Cooperation Using Team-mate Modeling and Joint Action Generalization", Adv. Sci. Technol. Eng. Syst. J. 5(2), 1-12, 2020. https://doi.org/10.25046/aj050201

[20] D. P. Kucherov, A. M. Kozub, "Mathematical model of UAV as agent of multi-agent system" in Dependable Systems, Services, and Technologies (DESSERT-2018), May 24-27, Kyiv, Ukraine, Proceedings, 358-362, 2018. https://doi.org/ 10.1109/DESSERT.2018.8409156

[21] D. Kucherov, O. Sushchenko, A. Rasstrygin, S. Zhdanov, A. Kozub, "Synthesis of the switching control law for a quadrotor autopilot", Int. J. of Eng. and Tech. (UAE), 7(4), 3065-3069, 2018. https://doi.org/10.14419/ijet.v7i4.16368

[22] B. Libet, Mind time: The temporal factor in consciousness, Perspectives in Cognitive Neuroscience, Harvard University Press, 2004.

[23] Dektak stylus capabilities. https://www.bruker.com/fileadmin/user_upload/8PDF-Docs/SurfaceAnalysis/StylusProfilometry/ApplicationNotes/AN526Dektak_Stylus_Capabilities_How_to_Choose_the_Corre.pdf

[24] A methodological approach to determining the influence of the human factor on the performance of information systems. (in Russian) https://habr.com/ru/company/cognitive/blog/209266/

[25] T. L. Saaty, "Relative Measurement and its Generalization in Decision Making Why Pairwise Comparisons are Central in Mathematics for the Measurement of Intangible Factors the Analytic Hierarchy/Network Process" Rev. R. Acad. Cien. Serie A. Mat., 102(2), 251 - 318, 2008. http://www.rac.es/ficheros/doc/00576.PDF

[26] A. A. Avetistova, "Psychological features of players in computer games", Psychology. J of the Higher School of Econ., 8 (4), 35 - 58, 2011. 